\title{
Meaning of The Fundamental Characteristics of Cancer in Tumorigenesis: Mini Review
}

\author{
Josenel Maria Barcelos Marçal ${ }^{1 *}$, Giuseppe Dick Bonato², Júlia Iaroseski² ${ }^{2}$ Lizandra Quandt ${ }^{2}$, \\ Andressa Gabriela dos Santos Lersch ${ }^{2}$, JoãoLins Maués ${ }^{2}$, Rafael Fabiano Machado Rosa ${ }^{3}$ and \\ Paulo Ricardo Gazzola Zen ${ }^{3}$
}

${ }^{1}$ Department of pathology and forensic medicine, Universidade Federal de Ciências da Saúde de Porto Alegre (UFCSPA), Brazil

2Graduation in Medicine, UFCSPA, Brazil

3Department of Internal Medicine, Clinical Genetics, UFCSPA and Irmandade Santa Casa de Misericórdia de Porto Alegre (ISCMPA), Brazil

*Corresponding author: Mrs. Josenel Maria Barcelos Marçal-UFCSPA/ISCMPA. Rua Sarmento Leite, 245/403-Bairro Centro. Porto Alegre, RS-Brasil.

To Cite This Article: Josenel Maria Barcelos Marça, Giuseppe Dick Bonato, Júlia Iaroseski, Meaning of The Fundamental Characteristics of Cancer in Tumorigenesis: Mini Review. Am J Biomed Sci \& Res. 2021 - 11(6). AJBSR.MS.ID.001697. DOI: 10.34297/AJBSR.2021.11.001697.

Received: 眥 February 08, 2021; Published: 制 February 12, 2021

\begin{abstract}
The theoretical framework on the fundamental characteristics of cancer and the tumor microenvironment has been the subject of many studies in recent years. Based on principles, several drugs were developed for the treatment of cancer, such as drugs that act on neoplastic cell metabolism, angiogenesis, apoptosis, genomic instability, among others center. In this review, we seek to provide, in a concise and objective way, data for understanding the process of cancer development and the resistance acquired by neoplastic cells.
\end{abstract}

Keywords: Cancer, Cancer biology, Brands, Malignancy, Therapies, Carcinogenesis

\section{Fundamental Characteristics of Cancer}

Cancer is a heterogeneous and complex disease at all levels (genetic, pathological, histological, prognostic and therapeutic), highly dynamic, with multiple molecular constituents in evolution. The emergence of a neoplasm occurs through the formation of transformed clones that generate diversified and selected subpopulations that acquire an increasing aggressiveness. The progression process occurs due to morphological, metabolic factors and changes in gene expression, caused by chromosomal abnormalities, point mutations and an aberrant DNA methylation status [1]. The accumulation of multiple changes leads the transformed cells to acquire typical characteristics and properties that provide advantages over their normal counterpart.

Hanahan and Weinberg [2] described six properties acquired from cancer during tumorigenesis: maintenance of cell proliferation, inhibition of tumor suppressor genes, cell immortality, evasion of apoptosis, sustained angiogenesis, activation of invasion and metastasis [2].

In 2011, the same authors described other characteristics of neoplasms, including the development and progression of cancer in inflammatory processes; genomic instability; alteration of energy metabolism (Warburg effect) and immune avoidance [3]. Knowledge about cancer genetics has progressed remarkably, with the development of sequencing techniques for genetic mutations. The cancer mutation scenario has been integrated with transcriptome, epigenome and metabolome maps [4]. From this knowledge therapeutic drugs have been developed for the treatment of cancer (Figure 1).

In normal cells, cell proliferation is an event controlled by antiproliferative signals or by induction of programmed cell death through an exogenous paracrine signal. 


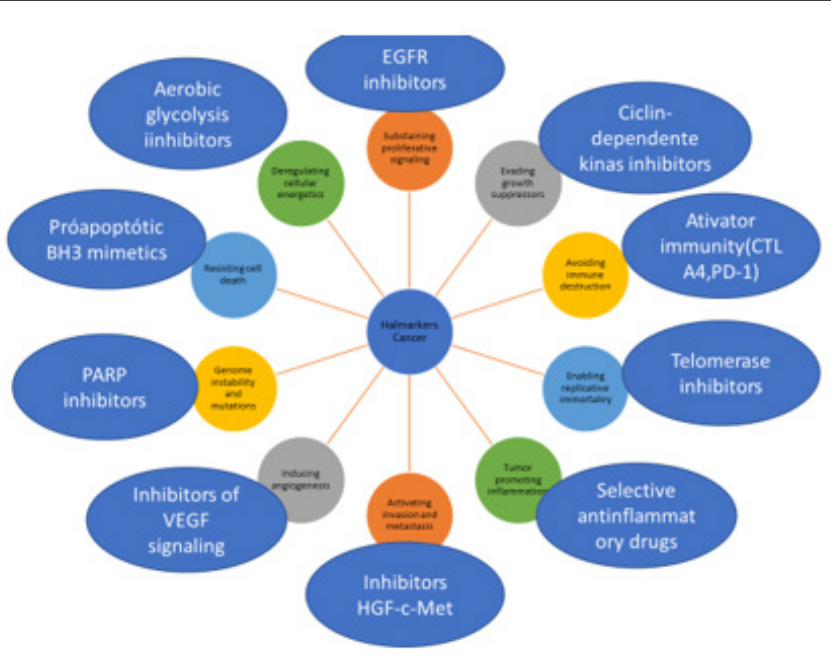

Figure 1: Therapeutic indications that act on each registered trademark of cancer [2].

In tumor cells, proliferation is mainly autonomous and decoupled from external mitogenic signals. Tumor cells synthesize growth factors through autocrine stimulation, causing the overexpression of a receptor that is more responsive to the normal concentration of ligand, or expressing constitutively activated surface receptors; or through the action of integrins (receptors that mediate cell matrix and cell-cell contact). Thus, they are able to increase cell survival, growth and maintenance of an aberrant regulation of the SOS-Ras-Raf-MAPK cascade that continues to transmit intracellular mitogenic signaling, even in the absence of normal upstream stimulatory signals [5].

During carcinogenesis, if the DNA is damaged, as well as if the nucleotides and nutrients are depleted, changes in the tumor suppressor RB (retinoblastoma) genes, which control entry into the cell cycle, may occur; and p53, which maintain the cell in the G1 / S phase of the cell cycle. Cancer cells use other strategies to escape programmed cell death, such as mutations that impair p53 function, with increased expression of apoptosis inhibitors ( $\mathrm{Bcl} 2$, $\mathrm{Bcl}-\mathrm{xL}$ ), negative regulation of pro-apoptotic factors (Bax, Bim and Puma) and the activation of the phosphatidylinositol 3-kinase (PI3K) -Akt6 survival pathway [5,6].

Tumor cells show unlimited replicative potential through the maintenance of telomerase or through a mechanism mediated by recombination, called ALT (alternative lengthening of telomeres) [7]. Angiogenesis occurs during the early stages of neoplastic progression. Endothelial cells are stimulated by endothelial growth factor-A (VEGF-A) and / or fibroblast growth factor (FGF) and negative regulation occurs through thrombospondin 1 (TSP1) or $\beta$-interferon8.

The new vessels contribute to the occurrence of metastases by the loss of expression or mutations of the E-cadherin gene, or by overloading genes that encode proteins involved in cell migration, such as N-cadherin8.

It is worth mentioning that some elements within the tumor microenvironment are important for the development of a neoplasm. These elements include parenchyma cells, such as fibroblasts, endothelial cells, pericytes, in addition to immune and inflammatory factors recruited from $\mathrm{T}$ and B cells, macrophages, neutrophils, mast cells and other cells derived from bone marrow. These groups of cells are responsible for the synthesis of noncellular components of the tumor-associated stroma, such as extracellular matrix proteins (ECM), proteases, cytokines and growth factors [9].

The tumor stroma fibroblasts secrete growth factors (HFG, EGF and b-FGF) and cytokines (SDF-1 and IL-6) leading to the infiltration of immune cells, which promote angiogenesis and metastatic spread; by ECM remodeling and, therefore, influencing the proliferation, survival and migration of cancer cells9. The Warburg effect is another property of the neoplastic cell that increases the energy potential with the production and secretion of lactate from glycogen, without using oxygen10. Infiltrating cells of the immune and inflammatory system include populations with a different and therefore functionally heterogeneous phenotype, mainly macrophages, dendritic cells (DCs), myeloidderived suppressor cells (MDSCs) and neutrophils. These cells can act in opposite ways, both antagonizing and promoting tumor development. Therefore, the frequency of inflammatory infiltration, as well as its stage of maturation and spatial location within the tumor microenvironment have decisive effects on the clinic and on the immune responses [11].

Other elements capable of affecting the behavior of malignant cells are of extracellular origin, such as $\mathrm{pH}$, oxygen concentration 
(02), tissue pressure and fluid flow [10]. The identification of the participation of these cells in tumor progression is paving the way for research and development of new therapeutic possibilities, targeting non-cancerous cells in the tumor microenvironment, which often do not have unregulated proliferation rates or increased genetic instability.

We cannot fail to mention that recent studies indicate that a carcinogenesis cannot be explained only by genetic changes, but also by epigenetic changes, such as DNA methylation, modifications of histones and microRNAs. These molecular changes lead to permanent changes in the patterns of gene expression that regulate the neoplastic phenotype [12].

Activated macrophages of type 1 (M1) secrete pro-inflammatory cytokines and chemokines, which lead the development and differentiation of auxiliary $\mathrm{T}$ lymphocytes 1 (Th1), Th17 and natural killer cells (NK). They have high microbicidal activity, immunostimulatory function and are present in incipient tumors, where they are capable of killing tumor cells13. Type 2 (M2) macrophages, on the other hand, express a set of different cytokines and chemokines, such as CCL17, CCL22 and CCL24, promoting the recruitment and development of regulatory $\mathrm{T}$ cells. This type of macrophage stimulates tissue repair and remodeling, the process of neoangiogenesis and tumor invasion and metastasis. Tumor cells can regulate the function of tumor-associated macrophages (TAMs), "educating" them for an M2phenotype [13-16].

Dendritic cells, derived from bone marrow, are antigen presenting cells (APCs) for T lymphocytes in lymphoid organs and tissues, and comprise two subpopulations: myeloid dentritic cells, which inhibit neoangiogenesis by producing IL-12 interleukin; and plasmocytoid dendritic cells, which induce angiogenesis through the production of TNF- @ and IL-8, as well as tumor progression and metastases through the secretion of proteases, such as MMP$1,-2,-3,-9$ and -19 and their inhibitors, called tissue inhibitor metalloproteinases (TIMP) -1 and -2 . These cells act as tumorpromoting cells, inhibiting innate and adaptive immunity and preventing the action of immunotherapy $[3,16]$.

Neutrophils are capable of promoting the process of angiogenesis, directly secreting VEGF, or inducing its release through the production of MMPs, but, like other inflammatory cells, they also have antitumor activity. In fact, they can kill tumor cells by releasing proteases, membrane-perforating molecules, reactive oxygen specimens (ROS) and cytokines, in particular TNF- $\alpha$ and IL-
$1 \beta$. According to the signals within the tumor microenvironment, it can inhibit angiogenesis by elastase production [3].

\section{Conclusion}

Tumorigenesis is a dynamic, multi-phase process, characterized by the acquisition of genetic and epigenetic alterations in which tumor cells acquire characteristics that maintain their survival and adaptation to the stress of the tumor microenvironment. Thus, the fundamental marks of cancer represent the basis for cancer research and the development of anti-neoplastic therapies.

\section{References}

1. Nowell PC (2000) Tumor progression: a brief historical perspective. Semin Cancer Biol 12(4): 261-266.

2. Hanahan D, Weinberg RA (2000) The hallmarks of cancer. Cell 100(1): 57-70.

3. Hanahan D, Weinberg RA. Hallmarks of cancer: the next generation. Cell 144(5): 646-674.

4. Moses C, Garcia-Bloj B, Harvey AR, Blancafort P (2018) Hallmarks of cancer: The CRISPR generation. Eur J Cancer 93:10-18.

5. Youssef A, Aboalola D, Han VK (2017) The Roles of Insulin-Like Growth Factors in Mesenchymal Stem Cell Niche. Stem Cells Int 2017: 9453108.

6. Evan G, Littlewood T (1998) A matter of life and cell death. Science 281(5381): 1317-1322.

7. Bryan TM, Cech TR (1999) Telomerase and the maintenance of chromosome ends. Current opinion in cell biology 11(3): 318-324.

8. Dameron KM, Volpert OV, Tainsky MA, Bouck N (1994) Control of angiogenesis in fibroblasts by $\mathrm{p} 53$ regulation of thrombospondin-1. Science 265(5178): 1582-1584

9. Lukashev ME, Werb Z (1998) ECM signalling: orchestrating cell behaviour and misbehaviour. Trends in cell biology 8(11): 437-441.

10. Pavlova NN, Thompson CB (2016) The Emerging Hallmarks of Cancer Metabolism. Cell Metab 23(1): 27-47.

11. De Palma M, Hanahan D (2012) The biology of personalized cancer medicine: facing individual complexities underlying hallmark capabilities. Mol Oncol 6(2): 111-127.

12. Chen QW, Zhu XY, Li YY, Meng ZQ (2014) Epigenetic regulation and cancer (review). Oncol Rep 31(2): 523-532.

13. Shields JD, Kourtis IC, Tomei AA, Roberts JM, Swartz MA (2010) Induction of lymphoidlike stroma and immune escape by tumors that express the chemokine CCL21. Science 328(5979): 749-752.

14. Hirata E, Sahai E (2017) Tumor Microenvironment and Differential Responses to Therapy. Cold Spring Harb Perspect Med 7(7): a026781.

15. Onuchic AC, Chammas R (2010) Câncer e o microambiente tumoral. Rev Med (São Paulo) 89(1): 21-31.

16. Coussens LM, Werb Z (2002) Inflammation and cancer. Nature 420(6917): 860-867. 